\title{
Design of Vacuum Compatible Automatic Targets Feeding System
}

\author{
Marian Curuia*, Sorin Soare, Catalin Jianu, Mihai Varlam \\ National Research and Development Institute for Cryogenic and Isotopic Technologies, Ramnicu Valcea, Romania \\ Email address: \\ marian.curuia@icsi.ro (M. Curuia), sorin.soare@icsi.ro (S. Soare), catalin.jianu@icsi.ro (C. Jianu), mihai.varlam@icsi.ro (M. Varlam) \\ ${ }^{*}$ Corresponding author
}

\section{To cite this article:}

Marian Curuia, Sorin Soare, Catalin Jianu, Mihai Varlam. Design of Vacuum Compatible Automatic Targets Feeding System. Nuclear Science. Vol. 3, No. 3, 2018, pp. 40-44. doi: 10.11648/j.ns.20180303.13

Received: October 4, 2018; Accepted: October 18, 2018; Published: November 14, 2018

\begin{abstract}
The present paper presents the design of a vacuum compatible automatic targets feeding system (VCATFS) that can be used to introduce targets inside the interaction chambers for accelerators, laser beams and others research plants. As a first application, the system is to be used within the Extreme Light Infrastructure - Nuclear Physics (ELI-NP) project, to load target frames in the interaction chamber without losing the vacuum. The target frames will be inserted and retracted into/from the interaction chamber individually with high accuracy of positioning. Up to three target frames can be used during one experiment. This number of targets is a consequence of several severe spatial constraints where the system will be installed, but for other situations it can be increased. Also, a new technique of moving the target frames is proposed, two horizontal translations in two parallel planes and two vertical translations also in two parallel planes. VCATFS is divided into two main sub-systems: mechanical (includes kinematics, high precision components and systems, vacuum chamber), and a dedicated command and control system (transducers - high accuracy absolute linear encoders, stepper motors and associated electric drives unit, interfaces and proprietary software). Additionally, a dedicated vacuum system was designed. This approach will further be developed as prototype level. This paper will focus on the design of mechanical sub-system, the remaining ones will be the subject of future documents that would be made publicly available in the future.
\end{abstract}

Keywords: Feeding System, Targets, Vacuum Compatible

\section{Introduction}

ELI-NP, one of the most advanced facility in the world, focused on the study of photonuclear physics and its applications consist of two $10 \mathrm{PW}$ ultra-short pulse lasers and one of the most brilliant tuneable gamma-ray beams. This unique experimental combination will enable ELI-NP to approach a wide range of research topics in fundamental physics, nuclear physics and astrophysics, and applied research in materials science [1]. Therefore, the maximisation of the beam time is a strong requirement. The strategy to meet this requirement was to design a vacuum compatible automatic target feeding system (VCATFS) that preserves the vacuum inside the interaction chamber, and that can work with a high number of target frames. Unfortunately, this last requirement is strongly limited by some spatial constraint of the place where VCATFS will be installed. The proposed design solution is able to supply up to three target frames between two successive load-lock openings.

Since the interaction chamber was already installed and in operation, the present on-site configuration placed heavy constraints on the design such as: laser beam position fixed, access points defined in terms of position size and shape, loading direction and technique, available space envelope and interfaces with both the interaction chamber and operator.

Taking into account the specific requirements regarding the number of target frames and the specific local configuration the translations had to be on both the vertical and horizontal axes: one vertical translation and two horizontal translation (two parallel planes).

\section{Concepts}

Several concepts have been evaluated and two were selected to be further developed at the conceptual design level. One of them is based on a linear feedthrough (Long 
Travel Linear Shift Mechanism - LTLS) [2] and the second on a rotary feedthrough (RF) [3], both for horizontal and vertical movements. For all approaches main requirements were to provide a vertical translation of $1205 \mathrm{~mm}$ and a horizontal translation of $+/-50 \mathrm{~mm}$, that is needed to reach the target frame final position (on the translation table of the goniometer inside the interaction chamber). For the horizontal movement there are no requested figures but considering the space envelope where the system is to be installed, the number and the dimensions of targets, a horizontal translation of $600 \mathrm{~mm}$ will be sufficiently.

Both approaches had to be dropped due to space envelope, especially on the vertical direction and a new solution based on a telescopic mechanism [4] was produced. However, the whole system, figure 1 , had to be designed as compact as possible to keep vacuum chamber volume within reasonable limits so that a medium-sized vacuum pump could be used within the budget. The ball screw feed drive concept served as base in the design for the horizontal unit [5].

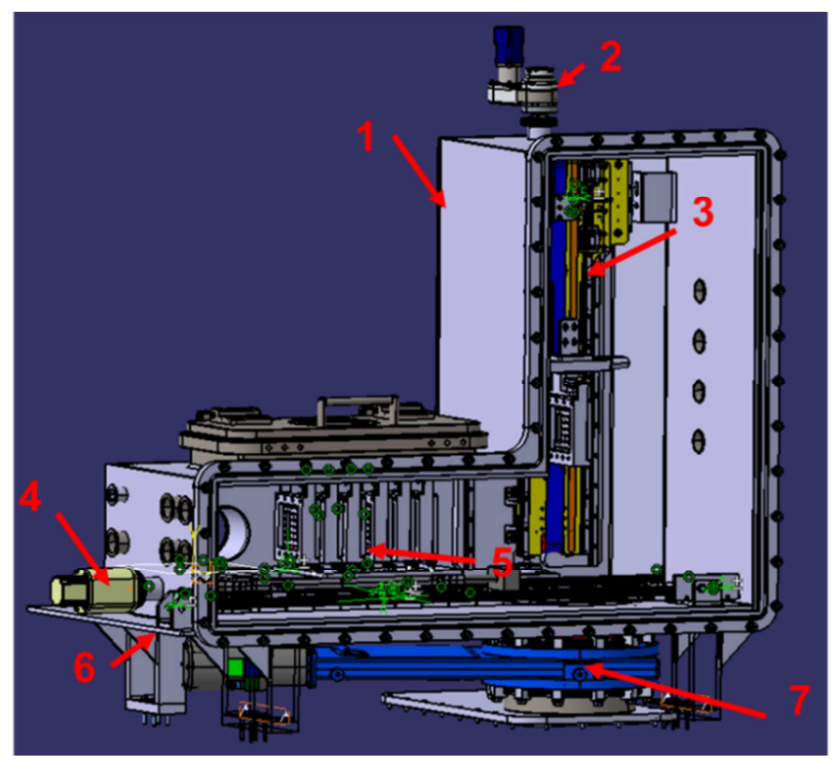

Figure 1. VCATFS, 1 - vacuum chamber, 2 - vertical drive unit, 3 telescopic arm, 4-horizontal drive unit, 5-horizontal platform with target frames, 6-vacuum chamber support, 7- high vacuum gate valve.

The vertical telescopic arm consists of three segments and with one driving unit. This driving unit uses the same ball screw feed drive concept as horizontal and is based on a DC stepper motor [6] placed outside the vacuum chamber. The movement is transferred to the telescopic arm by means a magnetic rotary feedthrough [3], all three segments move one relative to the other.

It is important to mention that for the first horizontal translation the same concept of driving unit [5] is used, with virtually no limitations in terms available horizontal space. The second horizontal translation, inside the VCATFS vacuum chambers, is based on a high vacuum motorised stage [7].

\subsection{Telescopic arm Design}

As mentioned before, the main constraint was the vertical available space. With all the components that have to be designed and installed, little space was left for the translation mechanism and the installation clearances. The solution chosen based on space availability, of-the-shelf components and costs was the one involving a belt transmission system [4], consisting of a metallic belt and two bearings.

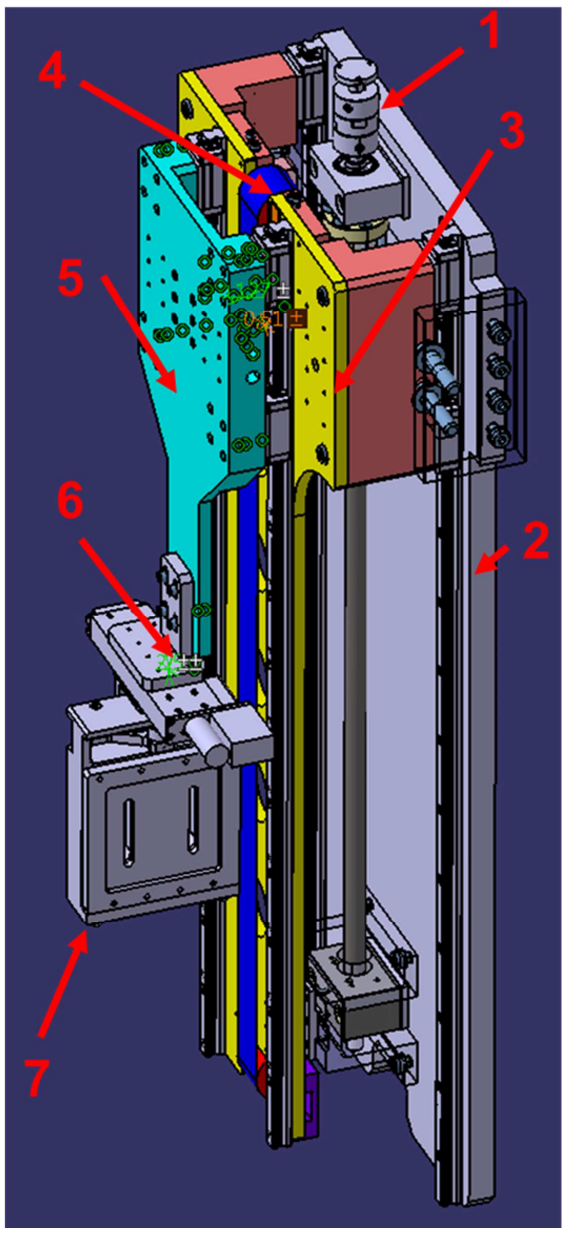

Figure 2. Belt transmission system, 1 -flexible coupling, 2 - first segment, 3 - second segment, 4 - belt, 5 - third segment, 6 - motorized vacuum stage, 7 - target frame.

Another advantage of this solution is that an external electric drive can be used thus allowing an easy maintenance and intervention for both vertical and horizontal translations.

The belt drives concept makes possible the translation of three segments with just on driving source while keeping the whole assembly compact. The kinematics behind this is the following: the belt with both ends clamped at one point to the first segment, wraps around two bearing bolted at the extremities of the second segment. The same belt is securely fixed (bolted) to the third segment. When the shaft [8] attached to the first segment is activated, via electric drive unit, the second segment begins its translation. In the same time the belt transfers the movement to the third segment which moves at a speed twice the speed of the first segment, 
figure 2. In this configuration, the belt acts as a movement amplifier and the bearings act as pulleys.

The segments are translating along two pairs of high precision linear guides bolted onto the first and second segments. The first segment position can be adjusted by using a push-pull system [9] at one end and a spherical rolling joint, figure 3 . This adjusting system uses the casing as rigid base and the final position as reference. The position adjusting unit are located at the extremities of the base plates (vertical and horizontal) as close as possible to the casing edges to prevent accidental positioning of target frame relative to the goniometer platform. The casing edges are the least features to be affected by deformation due to vacuum [10].

The motorized vacuum stage (MVS) is used to:

- places / takes the target frame on / from the horizontal table inside the VCATFS vacuum chamber

The target frames resting places are fitted with kinematic couplings, i.e. horizontal table (six positions) and goniometer platform (one position);

- places / takes the target frame on / from the goniometer platform inside the interaction chamber.

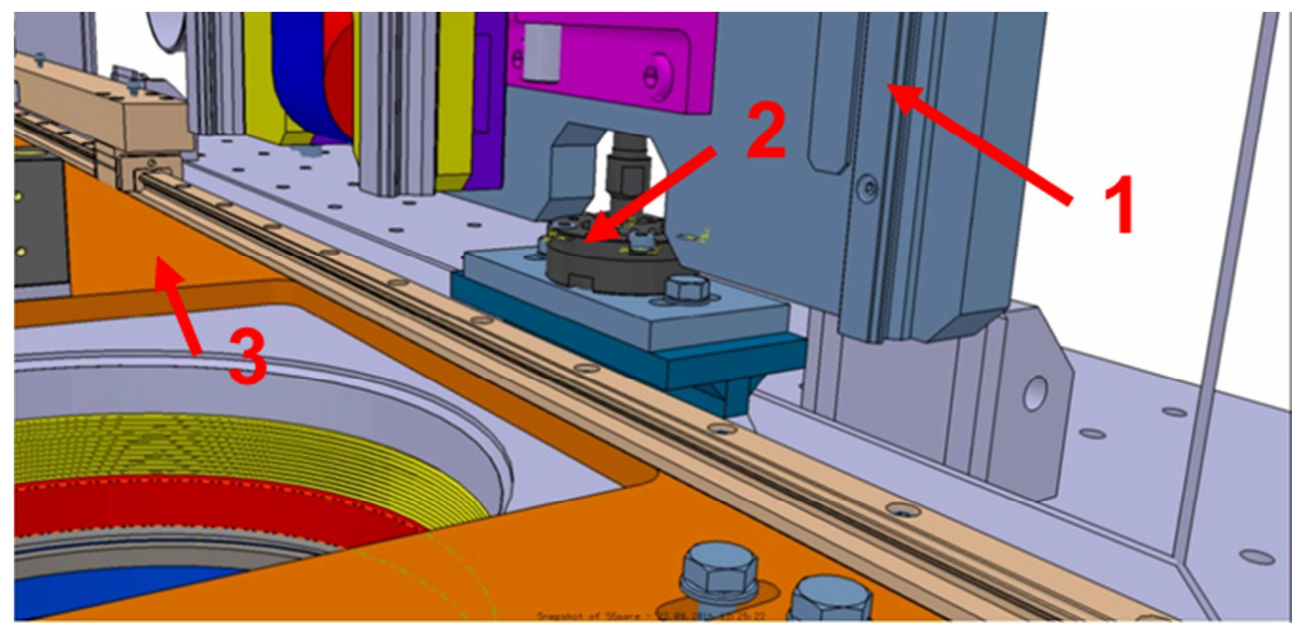

Figure 3. The adjusting system: spherical rolling joint used in conjunction with the push-pull system, 1 - first segment telescopic arm, 2 - spherical rolling joint, 3 - horizontal platform.

\subsection{Kinematics of VCATFS}

The kinematics of the VCATFS refers to all necessary motions [11] to transfer the target frame between the resting place from the horizontal platform till the positioning goniometer platform, inside the interaction chamber, figure 4. A special transfer device, attached to the MVS, takes the target frame from the horizontal platform and deposes it on the goniometer platform.

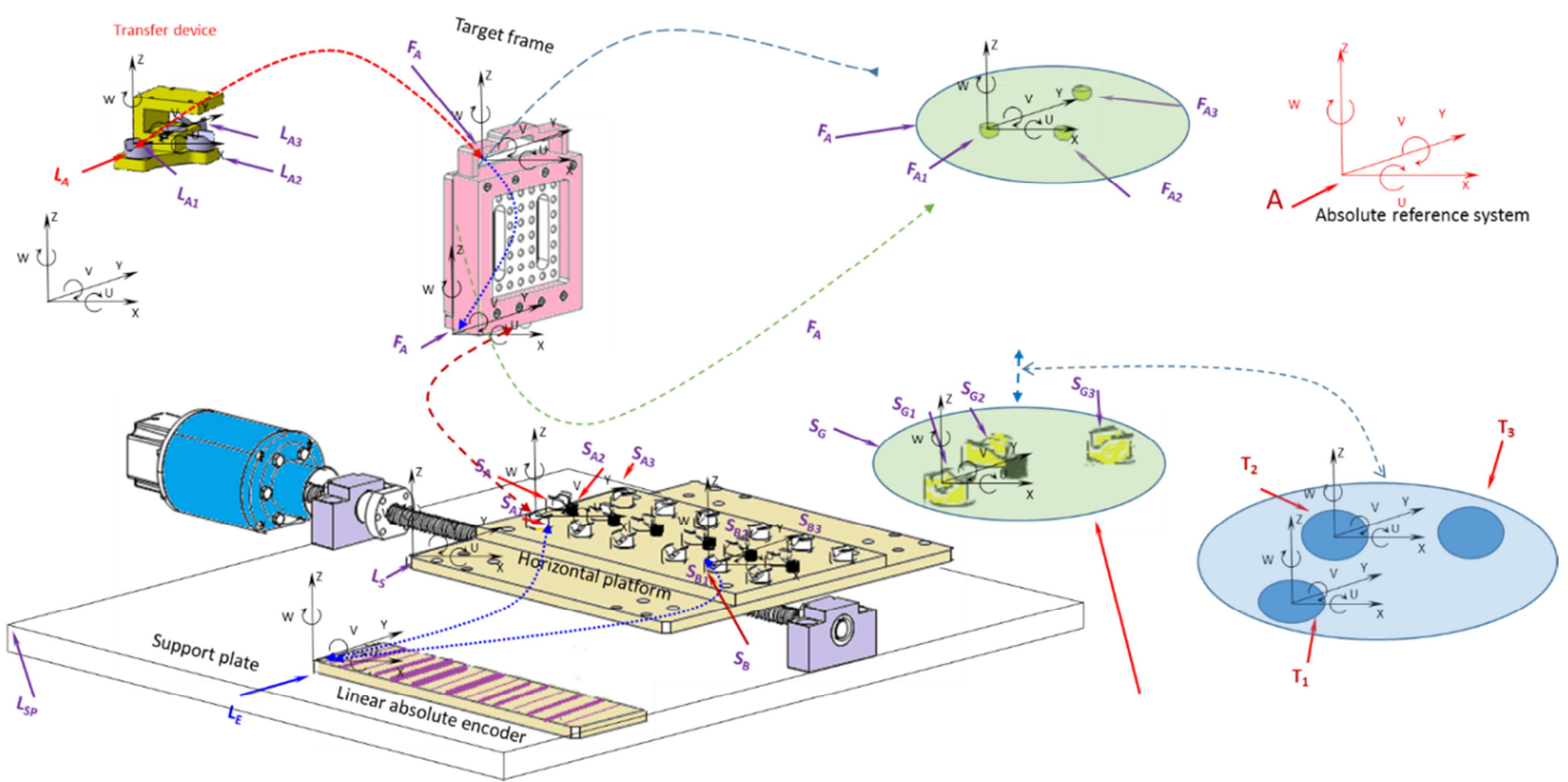

Figure 4. Kinematics of VCATFS. 
On the transfer device an interface $\mathrm{L}_{\mathrm{A}}$ that is made of three kinematic couplings, canoe type has been used. On the target frame another interface $F_{A}$, that consist of three truncated balls, has been introduced. The two interfaces form a high precision kinematic coupling that provides a very good accuracy in positioning of target frames. VCATFS has three high precision cinematic couplings $[12,13]$, the first, $L_{A}-F_{A}$, between transfer device and target frame, when the target frame is lifted, the second, $\mathrm{S}_{\mathrm{G}}-\mathrm{F}_{\mathrm{A}}$, between the target frame base and goniometer platform, and the third, $\mathrm{L}_{\mathrm{A}}-\mathrm{S}_{\mathrm{A}}$, between the target frame base and horizontal platform.

High precision kinematic coupling is very important but not sufficiently for obtaining a good accuracy in positioning of target frames, a very good precision is required for machining operation of all VCATFS components.

The kinematic scheme of VCATFS, to pick the target frame from the horizontal platform and subsequently to release it on the goniometer platform is shown in figure 5. For target frame OUT the kinematic scheme is somewhat similar with those for target frame IN.

The values of the figures are described in the table 1 .

The engagement between $\mathrm{L}_{\mathrm{A}}$ and $\mathrm{F}_{\mathrm{A}}$, is done in the following sequence:

1. A translation of $15 \mathrm{~mm}$ done by horizontal motorized stage, $x 3$ axis of the reference system solidary with this stage;

2. A movement of $5 \mathrm{~mm}$ on $\mathrm{z} 2$ axis of the reference system solidary with telescopic arm.

Thanks to the signal provided by the linear absolute encoders [14], the control system receives the exact position of the horizontal translation stage both for $\mathrm{x} 3$ and $\mathrm{z} 2$ axis. To avoid any false information related to the target engagement, one more information would be provided. A force sensing resistor could be a solution for this issue.

3. A movement with $145 \mathrm{~mm}$ on $\mathrm{z} 2$ axis to lift the target so that to permit the movement of horizontal platform on $\mathrm{x} 1$ axis.

The positioning between $\mathrm{L}_{\mathrm{A}}$ and $\mathrm{F}_{\mathrm{A}}$ is done by the absolute linear encoder. The repeatability is done by the $\mathrm{L}_{\mathrm{A}}$ and $\mathrm{F}_{\mathrm{A}}$ (kinematic coupling) repeatability [15].

The repeatability when the target is placed on the horizontal stage is done by the added values for the two kinematic couplings $S_{G}$ and $F_{A}$, respectively $30 \mu \mathrm{m}$ for each kinematic coupling.
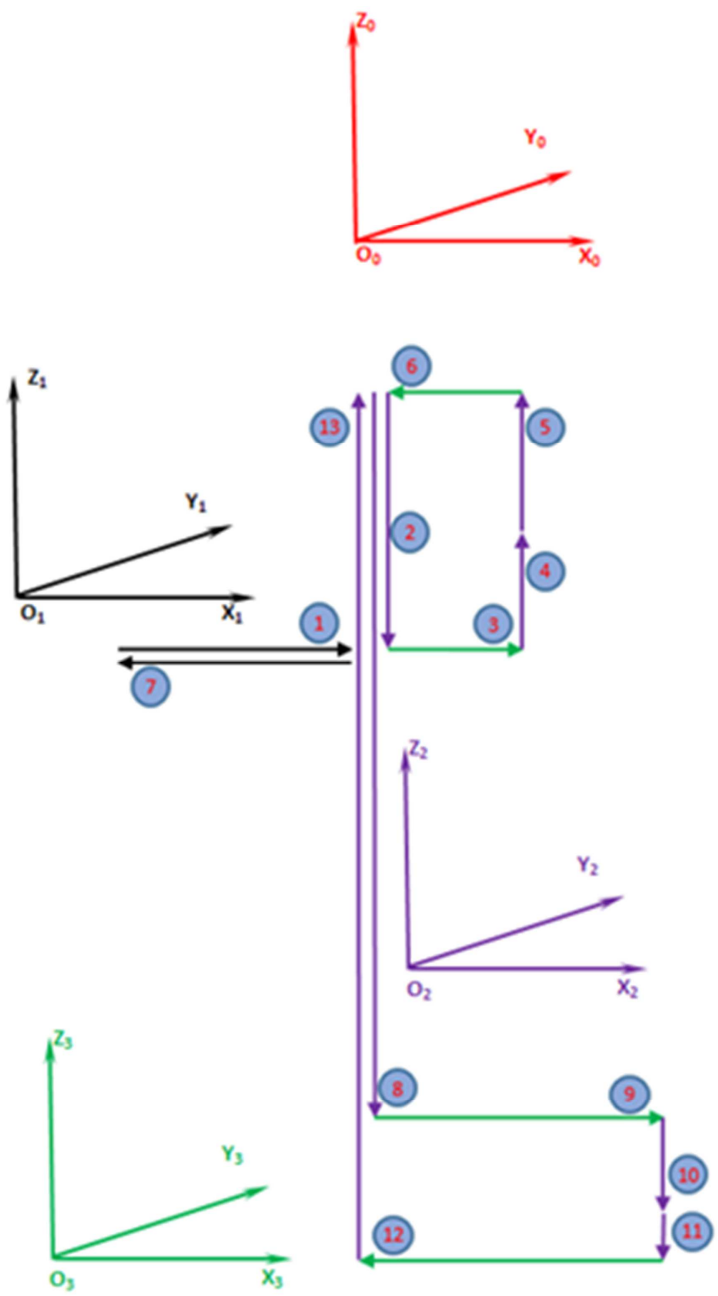

Figure 5. Kinematic scheme of VCATFS for target frame IN.

Table 1. The repeatability values for different parts of VCATFS.

\begin{tabular}{llllll}
\hline Target IN & & & & \\
\hline No. & System Axis & Comments & Sign/Distance (mm) & Repeatability (microns) & Repeatability Source \\
\hline 1 & X1 & Horizontal Platform & 445 & 5 & Linear Absolute Encoder \\
2 & Z2 & Telescopic arm & -150 & 5 & Linear Absolute Encoder \\
3 & X3 & Horizontal Motorized Stage & 15 & 2 & Stage Repeatability \\
4 & Z2 & Telescopic Arm & 5 & 35 & Linear Absolute Encoder + Kinematic \\
5 & Z2 & Telescopic Arm & 145 & 5 & Repeatability \\
6 & X3 & Horizontal Motorized Stage & -15 & 2 & Linear Absolute Encoder \\
7 & X1 & Horizontal Platform & -445 & 5 & Stage Repeatability \\
8 & Z2 & Telescopic Arm & -1150 & 2 & Linear Absolute Encoder \\
9 & X3 & Horizontal Motorized Stage & 50 & 5 & Linear Absolute Encoder \\
10 & Z2 & Telescopic Arm & -50 & 35 & Stage Repeatability \\
11 & Z2 & Telescopic Arm & -5 & 2 & Linear Absolute Encoder Absolute Encoder + Kinematic \\
12 & X3 & Horizontal Motorized Stage & -50 & 5 & Repeatability \\
13 & Z2 & Telescopic Arm & 1205 & 113 \\
Total & & & Stage Repeatability & Linear Absolute Encoder \\
\hline
\end{tabular}




\section{Conclusions}

In this paper a solution for VCATFS has been proposed and developed at detailed design stage from mechanical point of view. Maximum three targets frame can be supplied between two successive load - lock openings. With small changes of the horizontal platform and no spatial constraints, the number of target frames can be increased.

The hart of VCATFS, telescopic arm, is an original solution of the team and represents a combination between ball screw feed drive and belt transmission system. The main advantage of this solution is that a one drive unit and one linear transducer is needed for the telescopic arm and also must be mentioned that the proposed solution multiplies the linear speed of vertical platform third segment in comparison with the first one, by a factor of two. To provide a good accuracy in positioning high precision cinematic couplings together high resolution linear absolute transducer have been used. The design provides the possibility of adjustment both for horizontal and vertical system in order to ensure the perpendicularity between vertical axis of the system and laser beam axis. For this reason, a large bellows of $250 \mathrm{~mm}$ diameter has been introduced between vacuum chamber and the high vacuum gate valve and flexible couplings were also introduced between rotary feed through and ball screws.

\section{References}

[1] C. A. Ur Extreme Light Infrastructure-Nuclear Physics: The Future is Bright at Extremes, Journal Nuclear Physics News, Volume 26, 2016 - Issue 4.

[2] Long Travel Linear Shift Mechanism, HLSML Series, UHV Design Ltd.
[3] Magnetic Rotary Drives UHV Design Ltd.

[4] Donghun Lee et al. Design and Application of a Wire-Driven Bidirectional Telescopic Mechanism for Workspace Expansion with a Focus on Shipbuilding Tasks, Advanced Robotics 25 (2011) 699-715.

[5] S. Frey et al. Expedient Modeling of Ball Screw Feed Drives, Stuttgart 2011, SimTech - Cluster of Excellence Pfaffenwaldring 7a 70569 Stuttgart.

[6] Advanced Micro Systems, Inc. Stepper Motor System Basics (Rev. 5/2010).

[7] Motorized Positioning Systems, Zaber Technologies.

[8] Fundamentals of machine design, Version 2 ME, IIT Kharagpur.

[9] Adjustment mechanism, Optomechanical engineering handbook, Ahmad A., Boca Raton CRC Press LLC 1999.

[10] Hauviller, Design rules for vacuum chambers, CERN, Geneva, Switzerland.

[11] Machines and Mechanisms, Applied Kinematic Analisys, Fourth Edition, David H. Myszka, Prentice Hall 2012.

[12] Design and Analysis of Kinematic Couplings for Modular Machine and Instrumentation Structures by Anastasios John Hart B. S. E. Mechanical Engineering (2000) University of Michigan.

[13] Slocum, Alexander. "Kinematic Couplings: A Review of Design Principles and Applications." International Journal of Machine Tools and Manufacture 50.4 (2010): 310-327.

[14] RESOLUTETM absolute optical encoder with BiSS serial communications. Data sheet L-9517-9356, 2010-2012 Renishaw PLC.

[15] Martin L. Culpepper, Carlos Araque and Marcos Rodriguez, Design of Accurate and Repeatable Kinematic Couplings, Massachusetts Institute of Technology, Cambridge, MA. 